\title{
Juan Friede y su búsqueda de El adelantado don Gonzalo Jiménez de Quesada
}

\author{
José Eduardo Rueda Enciso \\ Escuela Superior de Administración Pública, Colombia
}

\begin{abstract}
Resumen
Se hace un detallado balance bibliográfico sobre las obras del historiador Juan Friede (1901-1990), que tratan sobre el adelantado Gonzalo Jiménez de Quesada, fundador de la ciudad de Santafé de Bogotá. También se hace una extensa revisión de las investigaciones que se han escrito sobre la vida y obra de este conquistador por parte de otros investigadores, señalando los aportes que cada uno ha realizado y la importancia de los trabajos de Friede como pionero del estudio de la conquista del Nuevo Reino de Granada y la problemática indígena colombiana, con base en la documentación guardada en el Archivo General de Indias (Sevilla), aprovechando una reciente reedición del libro El adelantado don Gonzalo Jiménez de Quesada (1979).
\end{abstract}

Palabras clave: JUAN FRIEDE, GONZALO JIMÉNEZ DE QUESADA, CONQUISTA, NUEVO REINO DE GRANADA, SIGLO XVI.

\begin{abstract}
Juan Friede's research on the adelantado Gonzalo Jimenez de Quesada, founder of Bogotá, is examined through a detailed bibliographic review. Taking advantage of a recent reprinting of Friede's book, El adelantado don Gonzalo Jiménez de Quesada (1979) an extensive examination of texts on the life of this conquistador by other researchers is also undertaken, pointing out their contributions and the importance of Friede's pioneering work on the conquest of the Kingdom of New Granada and the problems of Colombia's indigenous population through his use of documents found in the Archivo General de Indias (Sevilla).

Key words: JUAN FRIEDE, GONZALO JIMÉNEZ DE QUESADA, CONQUEST, NUEVO REINO DE GRANADA, $16^{\mathrm{TH}}$ CENTURY.
\end{abstract}


El periplo intelectual de Juan Friede Alter (Wlava, Ucrania, 1901 -Bogotá, Colombia, 1990; nacionalizado colombiano en 1930) es sorprendente, pues, de origen judío, luego de acumular una considerable fortuna como comerciante y representante de casas europeas (entre 1927 y 1935), concesionario de la Ford Motors Company en Manizales y Bogotá (entre 1935 y 1941), galerista de arte en Bogotá (el primer marchant que hubo en el país, entre 1940 y 1942) y tratante de ganado en San Agustín (1942-1947), a los cuarenta y dos años comenzó su carrera, que le ocupó el resto de su vida, de investigador del pasado, especialmente de los indígenas, un sector de la sociedad colombiana muy olvidado por la historiografía hasta ese momento, y en particular el proceso de aculturación, deculturación y transculturación que afrontaron las comunidades nativas al rozarse, contactarse y chocar con los españoles y europeos en cerca de tres siglos y medio de conquista y colonización de América. Con lo que se convirtió, junto con Luís Eduardo Nieto Arteta, Guillermo Hernández Rodríguez, Luís Ospina Vásquez y Jaime Jaramillo Uribe, en uno de los grandes pioneros de la moderna historiografía de Colombia.

La obra de Friede es extensa, abarca 300 títulos: 22 libros, 26 compilaciones documentales, artículos y ensayos, prólogos para reediciones, reseñas bibliográficas, ponencias en eventos internacionales; en su mayoría publicados en castellano, pero también en ingles, francés, alemán y cirílico. Algunos de sus libros, como El indio en lucha por la tierra. Historia de los resguardos del macizo central colombiano (1944, 1972 y 1976) ${ }^{1}$; Bartolomé de Las Casas (1475-1566). Su lucha contra la opresión $(1978)^{2}$; Los quimbayas bajo la dominación española. Estudio documental (1539-1810) (1963-1978) ${ }^{3}$; La otra verdad. La Independencia americana vista por los españoles $(1971,1972,1979)^{4}$; Los andaki, 1538-1947. Historia de la aculturación de una tribu selvática (1953, 1974) $)^{5}$, y Problemas sociales de los arhuacos: tierras, gobierno, misiones (1963-1973) ${ }^{6}$, superaron la primera edición y constituyen hitos importantes dentro de las temáticas allí abordadas.

\footnotetext{
${ }^{1}$ Juan Friede, El indio en lucha por la tierra. Historia de los resguardos del macizo central colombiano (1944; Bogotá: Punta de Lanza, 1976).

${ }^{2}$ Juan Friede, Bartolomé de Las Casas (1475-1566). Su lucha contra la opresión (Bogotá: Carlos Valencia, 1978).

3 Juan Friede, Los quimbayas bajo la dominación española. Estudio documental (15391810) (Bogotá: Carlos Valencia, 1978).

4 Juan Friede, La otra verdad. La Independencia americana vista por los españoles (Bogotá: Carlos Valencia, 1979).

${ }^{5}$ Juan Friede, Los andaki, 1538-1947. Historia de una aculturación de una tribu selvática (México: Fondo de Cultura Económica, 1953).

6 Juan Friede, Problemas sociales de los arhuacos: tierra, gobiernos, misiones (Bogotá: Universidad Nacional de Colombia, 1963).
} 
El libro El adelantado don Gonzalo Jiménez de Quesada (1979) ${ }^{7}$ es el cierre de una investigación que inició Friede en 1950 y que duró 29 años. La mayor parte de ese rastreo lo realizó, mientras que adelantaba, en el Archivo General de Indias de Sevilla, para la Academia Colombiana de Historia, entre 1947 y 1955, la recopilación documental de los diez tomos de Documentos inéditos para la historia de Colombia (1509-1550) (1955-1960) ${ }^{8}$ y en posteriores estadías del autor en los archivos españoles que se extendieron hasta 1978. Otros archivos visitados por Friede en búsqueda del rastro de Jiménez fueron: el de la ciudad de Córdoba en España, y en Bogotá, el antiguo Archivo Nacional de Colombia, hoy Archivo General de la Nación; sin embargo, el mayor cúmulo de información proviene del General de Indias. Trabajo editorial y de investigación sobre Jiménez de Quesada, al respecto del cual, Julián Ruiz Rivera dice:

... lo más importante que se ha producido [sobre el fundador de Bogotá] es el trabajo de recopilación documental y de interpretación histórica llevado a cabo por Juan Friede. Con todo, la sensación que queda es la de saber muy poco del personaje, pese a la cantidad de documentos que ha recopilado Friede, porque la mayor parte de esos documentos tienen carácter procesal y oficial ${ }^{9}$.

En buena parte, la investigación de El adelantado es simultánea y contemporánea, prima hermana, si se quiere, del libro Los chibchas bajo la dominación española $(1974)^{10}$. Efectivamente, además de la temática, pues Jiménez conquistó a los muiscas, en ambos encontramos algunas de las características propias de la producción intelectual de Friede: un riguroso y paciente rastreo documental acompañado de la escritura de avances convertidos en artículos, y por lo menos un libro de primera versión que luego fue corregido, aumentado y mejorado en por lo menos una segunda versión. Así, el artículo de arranque fue "Antecedentes histórico-geográficos del descubrimiento de la meseta muisca por el licenciado Gonzalo Jiménez de Quesada" publicado en 1950 en la Revista de Indias de Madrid $^{11}$. En 1951, con el apoyo del Consejo Superior de Investigaciones Científicas de España, en su colección Miscelánea Americana, editó dos volúmenes de documentos y ensayo con el mismo título, con los que definió las dos vertientes de trabajo anteriormente reseñadas pero también el interés por otros

\footnotetext{
${ }^{7}$ Juan Friede, El adelantado don Gonzalo Jiménez de Quesada (Bogotá: Carlos Valencia, 1979).

${ }^{8}$ Juan Friede, Documentos inéditos para la historia de Colombia, 10 t. (Bogotá: Academia Colombiana de Historia, 1955-1960).

9 Julián Ruiz Rivera, "De conquistador a colonizador: perfil antiheroico de Jiménez de Quesada", en Memorias del Congreso de Historia del Descubrimiento. Sevilla: 1992 (Sevilla, 1992), 2: 581-582.

${ }^{10}$ Juan Friede, Los chibchas bajo la dominación española (Bogotá: La Carreta, 1974).

11 Juan Friede, "Antecedentes histórico-geográficos del descubrimiento de la meseta chibcha por el licenciado Gonzalo Jiménez de Quesada", Revista de Indias (Madrid), 10, núm. 40 (1950): 327-348.
} 
protagonistas de la Conquista: los sacerdotes, los cronistas y especialmente el conquistador alemán, delegado de la casa de los banqueros Welser, Nicolás de Federmán, sobre los que Friede investigó importantes obras ${ }^{12}$.

A partir de 1951 hasta 1955, publicó 13 artículos, a manera de avance, sobre Jiménez, el descubrimiento, etc. Seis de ellos vieron la luz en las Lecturas Dominicales de El Tiempo, pues Friede contó siempre con el respaldo de las directivas del principal periódico del país y muy especialmente de su dueño, el ex presidente Eduardo Santos. En 1955, bajo el sello editorial de la desaparecida editorial Tercer Mundo, apareció el libro: Invasión del país de los chibchas, conquista del Nuevo Reino de Granada y fundación de Santafé de Bogotá: reevaluaciones y rectificaciones ${ }^{13}$. Siguió publicando artículos y ensayos relacionados, siete en total, hasta 1959, cuando para la Revista de Indias, números 77 y 78, publicó el artículo "El 450 aniversario del nacimiento de Gonzalo Jiménez de Quesada"14 que puede ser el primer esfuerzo por sistematizar lecturas de archivo, fuentes secundarias y algunos artículos escritos, a los que nos referiremos más adelante, sobre el adelantado y que tuvieron su primer gran publicación, por parte de la Academia Colombiana de Historia, en 1960: Gonzalo Jiménez de Quesada a través de documentos históricos. Estudio biográfico 1509-1550 $0^{15}$, que solo abarcó los primeros 41 años de vida de Jiménez, tiene una sólida base documental de 125 documentos y "ofrece a los historiadores, sin entrar en polémicas, nuevos datos sobre la vida del licenciado Jiménez de Quesada, los

\footnotetext{
12 Acerca de sacerdotes, son importantes sus trabajos sobre don Juan del Valle, primer obispo de Popayán: Juan Friede, Don Juan del Valle, primer obispo de Popayán (Segovia: Instituto Diego de Colmenares, 1952); "Breve reseña biográfica del segoviano Juan del Valle", Bolivar (Bogotá), 19 (mayo de 1953): 708-723; "Juan del Valle, primer obispo de Popayán y su formación indigenista”, Boletín de Historia y Antigüedades (Bogotá), 555556 (enero-febrero de 1961): 62-70. También, artículos, ediciones y prólogos de los cronistas coloniales fray Pedro Aguado, Recopilación historial, 4 t. ([c. 1574]; Bogotá: Presidencia de la República, 1956) y fray Pedro Simón, Noticias historiales de las conquistas de Tierra Firme en las Indias Occidentales, 7 t. ([¿1628?]; Bogotá: Banco Popular, 1981). Sobre la casa de los banqueros judío-alemanes de los Welser, está su monumental libro Los Welser en la conquista de Venezuela (Caracas: Edime, 1961). Sobre Nicolás de Federmán, está la traducción que en 1958 hizo de Historia indiana (Bogotá: Carco, 1958) y la biografía Vida y viajes de Nicolás de Federmán; conquistador, poblador y cofundador de Bogotá 1506-1542 (Bogotá: Buchholz, 1960).

${ }_{13}$ Juan Friede, Invasión del país de los chibchas, conquista del Nuevo Reino de Granada y fundación de Santafé de Bogotá: reevaluaciones y rectificaciones (Bogotá: Tercer Mundo, 1955).

14 Juan Friede, "El 450 aniversario del nacimiento de Gonzalo Jiménez de Quesada", Revista de Indias (Madrid), 19, núms. 77-78 (1959): 579-582.

${ }^{15}$ Juan Friede, Gonzalo Jiménez de Quesada a través de documentos históricos. Estudio biográfico 1509-1550 (Bogotá: ABC, 1960).
} 
cuales frecuentemente contradicen lo relatado por los cronistas coloniales, cuyas obras, sin embargo, deben tenerse en cuenta para la biografía de nuestro héroe"16.

En ese mismo año de 1960, en las prensas del Banco de la República, apareció Descubrimiento del Nuevo Reino de Granada y fundación de Bogotá (1536-1539). Según documentos del Archivo General de Indias, Sevilla. Revelaciones, rectificaciones ${ }^{17}$, que fue la segunda versión, aumentada y corregida, del libro aparecido en 1955. La aparición de esos dos libros en el mismo año correspondió a otra estrategia que tuvo Friede: para cada efemérides patria preparaba un libro relacionado con el tema: en 1960 se cumplían 150 años del grito de independencia y don Juan tuvo la disciplina intelectual de investigar y escribir no solo un libro sino cuatro, de gran impacto en el mundo intelectual de ese momento, pues, además de los libros de Jiménez y el Descubrimiento, publicó Los gérmenes de la emancipación americana en el siglo XVI, en la serie Monografías Sociológicas, No. 5, que editaba la Facultad de Sociología de la Universidad Nacional de Colombia $^{18}$, y Vida y viajes de Nicolás de Federmán: conquistador, poblador y cofundador de Bogotá 1506-1542, con el apoyo del librero Karl Buchholz ${ }^{19}$. Subrayando que ese año fue uno de los más prolíficos de su producción intelectual pues se publicaron un total de 12 títulos, que incluyen los cuatro libros reseñados y ocho artículos.

Durante la década del sesenta, la producción intelectual de Friede se amplió a otras temáticas, algunas correlacionadas con Jiménez, que ya hemos reseñado, y otras nuevas: Los quimbayas; La historia de Pereira ${ }^{20}$, así como temas de palpitante historia social, como los encomenderos, la formación de la capa social dirigente, la Independencia, etc. Entre 1960 y 1966, sólo publicó tres artículos relacionados con las primeras temáticas, pero en 1966 apareció la segunda edición de Invasión del país de los chibchas ${ }^{21}$. Igualmente, la década del setenta vio crecer su interés por fray Bartolomé de Las Casas y en 1974 y 1979 aparecieron las versiones últimas del descubrimiento: Los chibchas bajo la dominación española $(1974)^{22}$ y El

\footnotetext{
${ }^{16}$ Ibídem, 9.

${ }^{17}$ Juan Friede, Descubrimiento del Nuevo Reino de Granada y fundación de Bogotá (15361539). Según documentos del Archivo General de Indias, Sevilla. Revelaciones, rectificaciones (Bogotá: Banco de la República, 1960).

${ }^{18}$ Juan Friede, Los gérmenes de la emancipación americana en el siglo XVI. Monografías Sociológicas 5 (Bogotá: Universidad Nacional de Colombia, 1960).

${ }^{19}$ Friede, Vida y viajes.

${ }^{20}$ Luis Duque Gómez, Juan Friede y Jaime Jaramillo, Historia de Pereira (Pereira: Club Rotario de Pereira, 1963).

${ }^{21}$ Juan Friede, Invasión del país de los chibchas, conquista del Nuevo Reino de Granada y fundación de Santafé de Bogotá: reevaluaciones y rectificaciones, 2 ed. (Bogotá: Tercer Mundo, 1966).

${ }^{22}$ Friede, Los chibchas.
} 
adelantado don Gonzalo Jiménez de Quesada (1979) ${ }^{23}$, publicado en la conmemoración de los cuatrocientos años de la muerte de el adelantado y conquistador de la meseta muisca, por Carlos Valencia Editores, con una edición de 500 ejemplares patrocinados por el Banco de la República.

El adelantado es un libro que se divide en dos partes: la primera, el estudio biográfico y la segunda, que contiene un total de 87 documentos procedentes de los archivos arriba relacionados, que sustenta la primera. El conjunto es un bien concebido libro que superó los esfuerzos de otros historiadores colombianos por biografiar al descubridor.

Efectivamente, los primeros intentos por dar a conocer la biografía de Gonzalo Jiménez de Quesada por parte de escritores e historiadores aficionados colombianos datan del cuarto centenario del Descubrimiento de América, en 1892, cuando Ramón Zapata publicó "Gonzalo Jiménez de Quesada" y Pedro María Ibáñez editó Ensayo biográfico de Gonzalo Jiménez de Quesada ${ }^{24}$. Más adelante, en 1897, don Vicente Restrepo publicó Apuntes para la biografia del fundador del Nuevo Reino de Granada, y vidas de los ilustres prelados, hijos de Santafé de Bogotá: Gonzalo Jiménez de Quesada, el Ilustrísimo Sr. D. Hernando Arias de Ugarte, el Ilustrísimo Sr. D. Lucas Fernández Piedrahita ${ }^{25}$. Durante el siglo XX, el primero en escribir sobre el fundador de Bogotá fue Joaquín Maldonado Plata, con Gonzalo Jiménez de Quesada (1913) ${ }^{26}$; en 1922, lo hizo Raimundo Rivas, con "Jiménez de Quesada: hombre de Estado"27, y un año después el mismo autor publicó Los fundadores de Bogotá (Diccionario biográfico). Estudio presentado al Segundo Congreso de Historia y Geografía Hispanoamericana, reunido en Sevilla en mayo de $1921^{28}$; más adelante, en 1927, publicó "Los compañeros de Quesada". En 1926, Manuel José Forero Contreras dio a la luz publica "Del campo histórico: la casa de Jiménez de Quesada"29 y, en 1928, Ernesto Restrepo Tirado publicó De

${ }^{23}$ Friede, El adelantado.

24 José María Ibáñez, Ensayo biográfico de Gonzalo Jiménez de Quesada (Bogotá: Imprenta de la Luz, 1892).

${ }^{25}$ Vicente Restrepo, Apuntes para la biografia del fundador del Nuevo Reino de Granada, $y$ vidas de los ilustres prelados, hijos de Santafé de Bogotá: Gonzalo Jiménez de Quesada, el Ilustrísimo Sr. D. Hernando Arias de Ugarte, el Ilustrísimo Sr. D. Lucas Fernández Piedrahita (Bogotá: A.M. Silvestre, 1897).

26 Joaquín Maldonado Plata, Gonzalo Jiménez de Quesada (Bogotá: Tipografía Colón, 1913).

${ }^{27}$ Raimundo Rivas, “Jiménez de Quesada: hombre de Estado”, El Gráfico (Bogotá), 12, núm. 609 (1922).

28 Raimundo Rivas, Los fundadores de Bogotá (Diccionario biográfico). Estudio presentado al Segundo Congreso de Historia y Geografia Hispanoamericana, reunido en Sevilla en mayo de 1921 (Bogotá: Imprenta Nacional, 1923).

${ }^{29}$ Manuel José Forero, "Del campo histórico: la casa de Jiménez de Quesada", El Gráfico (Bogotá), 15, núm. 778 (abril 1926): 1421-1423. 
Gonzalo Ximénez de Quesada a don Pablo Morillo: documentos inéditos sobre la historia de la Nueva Granada ${ }^{30}$. En 1931, Enrique Otero D'Costa dio a la luz pública Gonzalo Jiménez de Quesada con el apoyo de la editorial Cromos ${ }^{31}$, libro este que fue investigado en cierto porcentaje en Sevilla, en 1910, cuando ese académico visitó el Archivo General. Para 1937, Alejandro Vallejo dio a conocer La cita de los aventureros: gesta de don Gonzalo Jiménez de Quesada ${ }^{32}$, que resistió una segunda edición en 1973 ${ }^{33}$. En 1939, Germán Arciniegas (1900-1999) lanzó Jiménez de Quesada ${ }^{34}$ que luego se convirtió, gracias a una corrección (tachaduras, enmiendas, reescritura) que el autor hizo de su propio puño y letra, en el libro original, en El caballero de El Dorado, el cual contó con una primera edición en 1940 y posteriores en 1942, 1950, 1969, 1970 y $1988^{35}$; dos ediciones (1942, 1943) en inglés: The Knight of El Dorado: the tale of Don Gonzalo Jiménez de Quesada and his conquest of New Grenada, now called Colombia ${ }^{36}$; una edición en rumano Kavalerul din El Dorado ${ }^{37}$ y otra en francés Le Chevalier d'El Dorado $^{38}$.

Tanto del libro de Otero D'Costa como del de Arciniegas, Friede escribió en el prologo del libro de 1960, Gonzalo Jiménez de Quesada a través de documentos históricos, que:

En los últimos años, los trabajos de Enrique Otero D'Costa y de algunos más, intentaron revaluar la personalidad de Jiménez, con base en una escasa documentación proveniente de fuentes dispersas. Otros, impulsados más por patriotismo que por el propósito de adelantar una verdadera investigación,

30 Ernesto Restrepo Tirado, De Gonzalo Ximénez de Quesada a don Pablo Morillo: documentos inéditos sobre la historia de la Nueva Granada (París: Le Moil, 1928).

${ }^{31}$ Enrique Otero D'Costa, Gonzalo Jiménez de Quesada (Bogotá: Cromos, 1931).

${ }^{32}$ Alejandro Vallejo, La cita de los aventureros: gesta de don Gonzalo Jiménez de Quesada (Bogotá: Litografía Colombia, 1938).

${ }^{33}$ Alejandro Vallejo, La cita de los aventureros: gesta de don Gonzalo Jiménez de Quesada (1938; Bogotá: Instituto Colombiano de Cultura, 1973).

${ }^{34}$ Germán Arciniegas, Jiménez de Quesada (Bogotá: ABC, 1939).

${ }^{35}$ Algunas ediciones de esta obra son: Germán Arciniegas, El caballero de El Dorado: vida del conquistador Jiménez de Quesada (1940; Buenos Aires: Lozada, 1942); El caballero de El Dorado (1940; Bogotá: Revista de Occidente, 1969); El caballero de El Dorado (México: Aguilar, 1978) y El caballero de El Dorado. Bogotá: Planeta, 1988.

${ }^{36}$ Germán Arciniegas, The Knight of El Dorado: the tale of Don Gonzalo Jiménez de Quesada and his conquest of New Grenada, now called Colombia (Nueva York: Viking, 1942).

37 Germán Arciniegas, Kavalerul din El Dorado, trads. Paul Alexandru Georgescu y Rosandra Maria Georgescu (Bucarest: Univers, 1987).

38 Germán Arciniegas, Le Chevalier d'El Dorado, trad. Georges Lomné (Montpellier, Francia: Espaces 34, 1995). 
ofrecieron panegíricos poco convincentes de la vida del conquistador, interpretando de modo antihistórico los pocos datos conocidos ${ }^{39}$.

Concepto que refinó Julián Ruiz Rivera cuando escribió que eran obras "de corte clásico y planteamiento literario" ${ }^{40}$.

Son contemporáneos a los libros de Friede sobre Jiménez de Quesada los trabajos de Luís Galvis Madero El adelantado $(1957)^{41}$, que es "una versión [relativamente] reciente de carácter sintético" 42 y de Humberto Villamarín Barrera Un conquistador (1968). En 1971, salió publicada una obra escrita probablemente a finales del siglo XIX, la de Soledad Acosta de Samper (1833-1913) titulada El descubridor y el fundador ${ }^{43}$.

Como efecto de los libros de Friede, dos historiadores extranjeros se preocuparon, en la década de los setenta, por el fundador de Bogotá: Demetrio Ramos Pérez publicó, con el apoyo del Consejo Superior de Investigaciones Científicas y la Escuela de Estudios Hispano-Americanos de Sevilla, el libro Ximénez de Quesada en su relación con los cronistas y el epitome de la conquista del Nuevo Reino de Granada $(1972)^{44}$, que se basa en el "Epítome de la conquista del Nuevo Reino de Granada" y entra en contradicción con Friede por la trascripción que don Juan hizo de ese documento, pero finalmente está de acuerdo con él en que sí fue Jiménez de Quesada el autor de "El epítome" y que es el mismo documento conocido como el "Gran cuaderno" que el licenciado y adelantado le prestó al cronista Gonzalo Fernández de Oviedo para escribir su crónica. El libro de Ramos es un exhaustivo análisis del "Epítome" y el mejor estudio critico sobre la faceta de cronista de Jiménez de Quesada.

Por su parte, el español Manuel Lucena Salmoral publicó "El indofeudalismo chibcha como explicación de la fácil conquista quesadista: consecuencias de una penetración por el Magdalena hacia la provincia del Metha" $(1975)^{45}$, que es una ponencia de 50 páginas presentada en el Simposio Hispanoamericano de

${ }^{39}$ Friede, Gonzalo Jiménez, 8.

${ }^{40}$ Ruiz, "De conquistador", 581.

${ }^{41}$ Luis Galvis, El adelantado (Madrid: Guadarrama, 1957).

${ }^{42}$ Ruiz, "De conquistador", 581.

43 Soledad Acosta de Samper, El descubridor y el fundador (Bogotá: Canal RamírezAntares, 1971).

${ }^{44}$ Demetrio Ramos, Ximénez de Quesada en su relación con los cronistas y el epítome de la conquista del Nuevo Reino de Granada (Sevilla: Escuela de Estudios HispanoAmericanos, 1972).

${ }^{45}$ Manuel Lucena Salmoral, "El indofeudalismo chibcha como explicación de la fácil conquista quesadista", en Estudios sobre política indigenista española en América, (Valladolid: Universidad de Valladolid, 1975), 1:111-160. 
Indigenismo Histórico, Terceras Jornadas Americanistas de la Universidad de Valladolid, centrada en la fase de cronista de Jiménez de Quesada, especialmente en el "Epítome de la conquista del Nuevo Reino de Granada" que ese autor transcribió en 1962 para la revista Ximénez de Quesada, del desaparecido Instituto de Cultura Hispánica, y reeditada en $1971^{46}$. Lucena utilizó de la producción de Friede la edición que este hizo de Aguado, los Documentos inéditos y Gonzalo Jiménez de Quesada a través de documentos históricos y aceptó la cronología que propuso sobre el conquistador. Tanto la obra de Ramos como la de Lucena son "estudios sobre su obra [la de Jiménez de Quesada] literaria e historica" "47.

Luego de la edición, en 1979, de El adelantado, es muy poco lo que se ha vuelto a publicar sobre Jiménez. En 1987, Manuel Ballesteros publicó Gonzalo Jiménez de Quesada ${ }^{48}$; en 1988, Manuel Lucena Salmoral editó Ximénez de Quesada: el caballero de El Dorado ${ }^{49}$, que, al igual que el libro de Galvis Madero $(1957)^{50}$, es "una versión reciente de carácter sintético",51. Otro español, Julián Bautista Ruiz Rivera, dio a la luz pública en 1992, con ocasión del quinto centenario del descubrimiento, la ya mencionada ponencia: "De conquistador a colonizador: perfil antiheroico de Jiménez de Quesada", un verdadero aporte al conocimiento de Jiménez, pues trabaja "un rasgo desconocido e importante del personaje, el de persona privada y ocupada en cuidar de sus escasos bienes y de saldar sus bienes"52, para lo que consultó un expediente procedente del Archivo Nacional de Bogotá (actual Archivo General de la Nación), Sección Colonia, Testamentarias de Cundinamarca, libro 13, folios 472-573, titulado: "Autos sobre los bienes de El adelantado, don Gonzalo Jiménez de Quesada", que trata de sus encomiendas y contiene las últimas dos cartas (2 de marzo y 7 de mayo) que escribió El adelantado antes de su muerte, en 1579.

En 1995, el colombiano, residente en los Estados Unidos, José Ignacio Avellaneda Navas publicó La expedición de Gonzalo Jiménez de Quesada al Mar del Sur y la creación del Nuevo Reino de Granada ${ }^{53}$, que es un libro muy bien investigado, en el que utilizó en extenso los materiales aportados por Friede, especialmente los diez tomos de Documentos inéditos, los ocho tomos de Fuentes documentales para

\footnotetext{
46 Manuel Lucena Salmoral, "Epítome de la conquista del Nuevo Reino de Granada, comentario y facsímil”, Ximénez de Quesada (Bogotá), 3, núm. 13 (1962): 33-60.

${ }^{47}$ Ruiz, "De conquistador", 581.

${ }^{48}$ Manuel Ballesteros, Gonzalo Jiménez de Quesada (Madrid: Historia 16; Quórum, 1987).

49 Manuel Lucena Salmoral, Ximénez de Quesada: el caballero de El Dorado (Madrid: Anaya, 1988).

${ }^{50}$ Galvis, El adelantado.

${ }^{51}$ Ruiz, "De conquistador", 581.

52 Ibídem, 582.

${ }^{53}$ José Ignacio Avellaneda, La expedición de Gonzalo Jiménez de Quesada al mar del sur y la creación del Nuevo Reino de Granada (Bogotá: Banco de la República, 1995).
} 
la historia del Nuevo Reino de Granada (1975-1976) ${ }^{54}$ y los dos tomos sobre Jiménez publicados en 1960; igualmente, utilizó las ediciones hechas por Friede de los cronistas Aguado y Simón y citó en extenso los dos tomos de El adelantado y el de Los Welser. Este autor tiene además dos importantes libros sobre los conquistadores que llegaron al territorio muisca: Los compañeros de Federmán cofundadores de Santa Fe de Bogotá (1990) y La expedición de Sebastián de Belalcázar al Mar del Norte y su llegada al Nuevo Reino de Granada $(1992)^{55}$.

En 1998, Enrique Santos Molano escribió Gonzalo Jiménez de Quesada que es una corta biografía de Jiménez destinada a un publicó no especializado ${ }^{56}$, especialmente el escolar, en la que utilizó también como fuente bibliográfica los trabajos de Friede pero también retoma la discutible biografía utilizada por Arciniegas en El caballero de El Dorado.

No podíamos dejar en este escrito sobre Juan Friede y Gonzalo Jiménez de Quesada sin una breve reseña sobre los aportes de Friede al conocimiento de la vida del licenciado, conquistador y adelantado. Sin embargo, en primer lugar, debemos hacer una referencia extensa al libro de Germán Arciniegas El caballero de El Dorado, pues a partir de él podemos entender el gran aporte que Friede hizo a la historiografía nacional con su cuidadoso trabajo de investigación y la edición de sus dos libros sobre Jiménez de Quesada. En realidad, buena parte de la producción intelectual de Friede se dedicó a repensar, investigar, reescribir y, en fin, a revisar y dar respuesta a algunas de las inconsecuencias e incongruencias sobre algunos temas históricos que Arciniegas escribió con la ligereza y diletancia que lo caracterizó o, como bien lo expresó el geógrafo Carl O. Sauer, "De Arciniegas he leído lo suficiente para considerarlo superficial y tendencioso. Lo que en general los colombianos necesitan es menos frases bonitas y más trabajo duro, ${ }^{\circ 7}$.

Apoyamos nuestra tesis de la respuesta investigativa que dio Friede a los temas que Arciniegas trató, basados en una carta que en junio de 1942 le envió al entonces Ministro de Educación Nacional, Germán Arciniegas, y que dice:

\footnotetext{
54 Juan Friede, Fuentes documentales para la historia del Nuevo Reino de Granada, 8 t. (Bogotá: Banco Popular, 1975-1976)

55 José Ignacio Avellaneda, Los compañeros de Federmán cofundadores de Santa Fe de Bogotá (Bogotá: Academia de Historia de Bogotá, Tercer Mundo, 1990); La expedición de Sebastián de Belalcázar al mar del norte y su llegada al Nuevo Reino de Granada (Bogotá: Banco de la República, 1992).

${ }^{56}$ Enrique Santos Molano, Gonzalo Jiménez de Quesada (Bogotá: Panamericana, 1998).

${ }^{57}$ Carl O. Sauer, "Informe de Carl O. Sauer a la Fundación Rockefeller sobre su visita a Colombia en 1942”, Estudios Sociales (Medellín), 1, núm. 3 (septiembre de 1988), 151.
} 
... siendo franco, ¿no podría un escritor europeo dotado de su talento y su erudición histórica y literaria, escribir Los estudiantes de la Mesa Redonda, América Tierra Firme o Los Comuneros? Es cierto que es Ud. uno de los mejores prosistas que tiene Colombia, si mi opinión algo vale. Lo considero además el único escritor colombiano que sabe hacer sentir al lector los acontecimientos históricos como cosas vivas, de la misma manera que en sus mejores libros Zweig, Maurois, Ludwig etc., pero no encuentro en sus libros una esencia americana, un elemento que sólo un americano sea capaz de sentir y escribir ${ }^{58}$.

En el caso de Jiménez de Quesada, don Germán publicó, en 1939, Jiménez de Quesada y la versión final en 1940 conocida como El caballero de El Dorado. El trabajo "fuerte" de la consulta para ese libro lo hizo Arciniegas en los cronistas coloniales: los frailes Pedro Aguado ${ }^{59}$ y Pedro Simón ${ }^{60}$; el sacerdote y versificador Juan de Castellanos ${ }^{61}$; los cronistas mayores Gonzalo Fernández de Oviedo ${ }^{62}$ y Antonio de Herrera ${ }^{63}$; el soldado-cronista Pedro Cieza de León ${ }^{64}$, los obispos Lucas Fernández de Piedrahíta ${ }^{65}$ y fray Bartolomé de Las Casas ${ }^{66}$, pues, en su concepto:

... constituyen la base principal en que naturalmente tiene que apoyarse quien trate asuntos de la conquista (...) No tratándose aquí de una obra de polémica, y siendo por lo general contradictorias las narraciones de los cronistas, en cada caso me he atenido a lo que el sentido común me ha indicado como lo más probable, haciendo los cotejos que la buena fe me ha dictado. No pretendo, además, haber escrito una biografía de Quesada, sino una breve historia de la conquista. Jiménez de Quesada

58 Juan Friede a Germán Arciniegas, Ministro de Educación Nacional, Bogotá, junio de 1942, en José Eduardo Rueda Enciso, "El pájaro caminante. Biografía de Juan Friede”, mecanografiado (Bogotá, s. f.), 170.

${ }^{59}$ Aguado, Recopilación historial.

${ }^{60}$ Simón, Noticias historiales.

${ }^{61}$ Juan de Castellanos, Elegías de varones ilustres de Indias ([¿1590-1592?]; Bucaramanga, Colombia: Gerardo Rivas Moreno, 1997).

${ }^{62}$ Gonzalo Fernández de Oviedo y Valdés, Historia general y natural de las Indias, islas y tierra firme del mar océano, 15 t. ([c. 1547]; Asunción, Paraguay: Guarania, 1944).

${ }^{63}$ Antonio de Herrera, Historia general de los hechos de los castellanos en las islas y tierra firme del mar océano, 17 t. (Madrid: Tipografía de Archivos; Maestre, 1934-1957).

${ }^{64}$ Pedro Cieza de León, La crónica del Perú (Bogotá: Ediciones de la Revista Ximénez de Quesada, 1971).

${ }^{65}$ Lucas Fernández de Piedrahíta, Historia general de las conquistas del Nuevo Reyno de Granada, 2 t. (Amberes, Holanda: Juan Baptista Verdussen, 1688; facsímil, Cali, Colombia: Carvajal, 1986).

${ }^{66}$ Fray Bartolomé de Las Casas, Historia de las Indias, 3 t. (México: Fondo de Cultura Económica, 1951). 
viene a ser la figura central, pero no he querido que su biografía oculte el resto de la historia ${ }^{67}$.

Los documentos originales que consultó Arciniegas fueron: el citado libro de don Enrique Otero D'Costa y una serie de documentos que don Ernesto Restrepo Tirado publicó en el Boletín de Historia y Antigüedades y un legajo de documentos inéditos consultados por Restrepo en los archivos de Sevilla, que se conservaban en la Academia de Historia y pasaron a formar parte del acervo documental del Archivo General de la Nación. Sobre dicho legajo, una autorizada voz, como es la de Jaime Jaramillo Uribe, opinó:

La Academia hizo un esfuerzo en los años 20 con Ernesto Restrepo Tirado. Él era un señor aficionado a la historia, como han sido todos los académicos aquí, que no sé por qué circunstancias fue a los archivos españoles y sacó de allá un mundo de cosas; pero los documentos que sacó no son utilizables porque están mal trascritos y sin criterios. El tipo no tenía entrenamiento, incluso en el hecho elemental de la trascripción y de la lectura paleográfica ${ }^{68}$.

No sobra decir que Friede desconfió mucho de las comisiones que en años pasados había encargado la Academia en Sevilla a otros académicos, pues habían sido muy limitadas y superficiales, y en muchos casos se había caído en lo anecdótico, en lo sensacionalista, como también en la búsqueda de documentación sobre héroes, dejando de lado los sectores populares y olvidados de nuestra sociedad. En su concepto, se había dejado de leer y recopilar mucho material, y aunque parece que, a propósito, él dejó de lado mucha documentación existente, es muy cierto que acopió bastante información que hasta ese momento no había sido tenida en cuenta por los historiadores de la Academia de Historia y que recopiló con el fín de reenfocar y desmitificar muchos aspectos de la historia de Colombia. Friede fue un conocedor profundo del Archivo General de Indias y un convencido de que "los estudios históricos deben basarse sobre documentos bien trascritos, desconfiando de copias defectuosas o descuidadas publicaciones" ${ }^{\text {"69 }}$.

En esencia, el libro de Arciniegas es interpretativo, inexacto y falto de objetividad, como lo fue la mayoría de su obra historiográfica, es decir, trató menos de revelar lo acontecido que de probar su postura personalista ante hechos que a veces sólo conoció superficialmente, por falta de una labor previa de investigación de los

${ }^{67}$ Germán Arciniegas, "Fuentes biográficas", en Jiménez de Quesada, 343. La copia que corrigió Arciniegas reposa en la Biblioteca Nacional de Colombia (Bogotá), Fondo Arciniegas, 5153. Resaltados del reseñador.

${ }^{68}$ Jaime Jaramillo Uribe, entrevista con el autor, Bogotá, febrero de 1990, en Rueda, "El pájaro caminante", 250.

69 Juan Friede al presidente y demás miembros de la Academia Colombiana de Historia, Bogotá, 19 de enero de 1954, Ibídem, 251. 
documentos históricos. De allí la importancia de los aportes de los dos libros investigados y escritos por Friede: en primer lugar, la documentación que aportó es, en un alto porcentaje, totalmente original, y la que quizás fue utilizada por otros historiadores, la consultó y transcribió en la fuente original. Los cronistas fueron examinados con visión crítica, de allí que en el transcurso del proceso de investigación de Jiménez publicó dos reediciones corregidas y aumentadas, con su prólogo respectivo de Aguado y Simón.

En segundo lugar, don Juan Friede colocó a Jiménez en un pedestal humano, mostrando sus cualidades y defectos en diferentes escenarios, como fue el de conquistador, encomendero, colonizador y jurista. Es así como, contradiciendo lo expresado por los cronistas, Friede demostró que Jiménez otorgó títulos de encomiendas, por los meses de abril y mayo de 1539, antes de emprender viaje a España, a varios de los conquistadores que lo acompañaron en la expedición descubridora, con lo que quiso darles los medios de subsistencia, y comprueba la preocupación del licenciado por la suerte de sus compañeros de aventura.

En tercer lugar, la base documental aportada por Friede (dos juicios de residencia contra Jiménez, visitas, infinidad de pleitos, probanza de servicios) es útil para reconstruir fragmentos de la historia administrativa del Nuevo Reino de Granada, de los primeros repartimientos de encomiendas, los procedimientos y rituales propios de la época, etc.

En cuarto lugar, don Juan dio a conocer aspectos olvidados y discutidos de la vida de El adelantado: su origen de judío converso, que sustentó en el expediente del pleito entre Lázaro Fonte y Jiménez de Quezada; el sumario de la petición de Alonso Luís de Lugo, según la cual Jiménez debía devolverle o traspasarle la Gobernación del Nuevo Reino de Granada; la denominación de La Tora, lugar donde El adelantado comenzó la subida a la meseta muisca, inicialmente llamado Pueblo de los Brazos, cuya voz no es indígena ni tampoco castellana, sino que corresponde al rollo pergamino en que los judíos tienen trascrito el Viejo Testamento que veneran y guardan en el altar de la sinagoga. En la Edad Media esta expresión se utilizaba, por antonomasia, para designar también el tributo que pagaba una comunidad judía. Lo que escribió el cronista Juan de Castellanos, testigo directo de muchos de los acontecimientos que relató, que al fundarse por primera vez Bogotá se construyeron 12 ranchos de paja en memoria de las doce tribus de Israel y no, como en el siglo XVII lo narró fray Pedro Simón, en homenaje a los 12 apóstoles. O el verdadero lugar y fecha de nacimiento de Jiménez de Quesada: la señorial Córdoba o la alegre Granada; en 1499 (la tradicional, calculada con base en los cronistas) o en 1509 (determinada por Friede, luego de paciente búsqueda, en declaraciones que el conquistador y su familia suministraron en pleitos y peticiones). 
Entre otros datos muy curiosos sobre la cotidianidad de la conquista, están: dificultades como la escasez de papel y tinta, indispensables para los españoles, dado su legalismo y costumbre de estampar todos sus actos y tratos en papel y ante escribano, y que fueron sustituidos por cuero de venado y sangre del mismo animal o por achiote. Muchos documentos de importancia para la reconstrucción de la naciente Colombia, como la repartición de las primeras encomiendas, fueron inicialmente escritos y se conservaron gracias a que, una vez solventada la escasez de papel, los interesados hicieron sacar por los escribanos copias autorizadas de los originales, antes que desaparecieran por virtud del endeble material en que estaban escritos. Las posibilidades de conservación física eran muy difíciles, normalmente se volvían ininteligibles en el transcurso de dos o tres años y la legibilidad de los mismos era igualmente dudosa. Sin embargo, Friede contó con muy buena suerte, pues a comienzos de los maravillosos años sesenta del siglo pasado, en 1962, cuando se encontraba en el Archivo de Indias, entre los legajos allí existentes encontró uno que probablemente nunca fue abierto y que contenía un original, que data de 1539 , de los que venimos refiriendo y que por hallarse prensado por las páginas del legajo, había resistido, un poco carcomido, más de cuatro siglos. El hallazgo hecho por Friede mereció un reconocimiento especial de las directivas del Archivo General de Indias y por años el precioso documento ocupó vitrina de honor.

Así, la iniciativa de Intermedio Editores por reeditar, en un volumen, El adelantado es importante porque las nuevas generaciones de historiadores colombianos y en general de estudiosos de las ciencias sociales y humanas tienen la oportunidad de conocer un excelente trabajo, no solo por su concepción sino por su cuidadosa investigación, difícil de encontrar en estos tiempos posmodernos en los que es común armar sobre un solo documento una narración histórica. Habida cuenta de que la obra misma de Friede constituye un hito dentro de la moderna historiografía nacional.

\section{Bibliografía}

Acosta de Samper, Soledad. El descubridor y el fundador. Bogotá: Canal RamírezAntares, 1971.

Aguado, fray Pedro. Recopilación historial. 4 t. [c. 1574]; Bogotá: Presidencia de la República, 1956.

Arciniegas, Germán. Jiménez de Quesada. Bogotá: Biblioteca Revista de las Indias, 1939. 
. El caballero de El Dorado: vida del conquistador Jiménez de Quesada. 1940; Buenos Aires: Lozada, 1942.

. The Knight of El Dorado: the tale of Don Gonzalo Jiménez de Quesada and his conquest of New Grenada, now called Colombia. Nueva York: Viking, 1942.

. El caballero de El Dorado. 1940; Bogotá: Revista de Occidente, 1969.

. El caballero de El Dorado. México: Aguilar, 1978.

Kavalerul din El Dorado, trads. Paul Alexandru Georgescu y Rosandra Maria Georgescu. Bucarest: Univers, 1987.

. El caballero de El Dorado. Bogotá: Planeta, 1988.

. Le Chevalier d'El Dorado, trad. Georges Lomné. Montpellier, Francia: Espaces 34, 1995.

Avellaneda, José Ignacio. Los compañeros de Federmán cofundadores de Santa Fe de Bogotá. Bogotá: Academia de Historia de Bogotá, Tercer Mundo, 1990.

. La expedición de Sebastián de Belalcázar al mar del norte y su llegada al Nuevo Reino de Granada. Bogotá: Banco de la República, 1992.

. La expedición de Gonzalo Jiménez de Quesada al mar del sur y la creación del Nuevo Reino de Granada. Bogotá: Banco de la República, 1995.

Ballesteros, Manuel. Gonzalo Jiménez de Quesada. Madrid: Historia 16; Quórum, 1987.

Castellanos, Juan de. Elegías de varones ilustres de Indias. [¿1590-1592?]; Bucaramanga, Colombia: Gerardo Rivas Moreno, 1997.

Cieza de León, Pedro. La crónica del Perú. Bogotá: Ediciones de la Revista Ximénez de Quesada, 1971.

Duque Gómez, Luis, Juan Friede y Jaime Jaramillo, Historia de Pereira. Pereira: Club Rotario de Pereira, 1963.

Federmán, Nicolás. Historia indiana. Trad. Juan Friede. Bogotá: Carco, 1958. 
Forero, Manuel José. "Del campo histórico: la casa de Jiménez de Quesada". El Gráfico (Bogotá), 15, núm. 778 (abril 1926): 1421-1423.

Friede, Juan. "Antecedentes histórico-geográficos del descubrimiento de la meseta chibcha por el licenciado Gonzalo Jiménez de Quesada". Revista de Indias (Madrid), 10, núm. 40 (1950): 327-348.

. Don Juan del Valle, primer obispo de Popayán. Segovia: Instituto Diego de Colmenares, 1952.

Los andaki, 1538-1947. Historia de una aculturación de una tribu selvática. México: Fondo de Cultura Económica, 1953.

. "Breve reseña biográfica del segoviano Juan del Valle". Bolívar (Bogotá), 19 (mayo de 1953): 708-723.

. Invasión del país de los chibchas, conquista del Nuevo Reino de Granada y fundación de Santafé de Bogotá: reevaluaciones y rectificaciones. Bogotá: Tercer Mundo, 1955.

. "El 450 aniversario del nacimiento de Gonzalo Jiménez de Quesada". Revista de Indias (Madrid), 19, núms. 77-78 (1959): 579-582.

- Documentos inéditos para la historia de Colombia, 10 t. Bogotá: Academia Colombiana de Historia, 1955-1960.

- Descubrimiento del Nuevo Reino de Granada y fundación de Bogotá (1536-1539). Según documentos del Archivo General de Indias, Sevilla. Revelaciones, rectificaciones. Bogotá: Banco de la República, 1960.

. Gonzalo Jiménez de Quesada a través de documentos históricos. Estudio biográfico 1509-1550. Bogotá: ABC, 1960.

Los gérmenes de la emancipación americana en el siglo XVI. Monografías Sociológicas 5. Bogotá: Universidad Nacional de Colombia, 1960.

- Vida y viajes de Nicolás de Federmán; conquistador, poblador y cofundador de Bogotá 1506-1542. Bogotá: Buchholz, 1960.

"Juan del Valle, primer obispo de Popayán y su formación indigenista". Boletín de Historia y Antigüedades (Bogotá), 555-556 (enero-febrero de 1961): 62-70. 
. Los Welser en la conquista de Venezuela. Caracas: Edime, 1961.

. Problemas sociales de los arhuacos: tierra, gobiernos, misiones. Bogotá: Universidad Nacional de Colombia, 1963.

. Invasión del país de los chibchas, conquista del Nuevo Reino de Granada y fundación de Santafé de Bogotá: reevaluaciones y rectificaciones. 2 ed. Bogotá: Tercer Mundo, 1966.

. Los chibchas bajo la dominación española. Bogotá: La Carreta, 1974.

. Fuentes documentales para la historia del Nuevo Reino de Granada. $8 \mathrm{t}$. Bogotá: Banco Popular, 1975-1976.

. El indio en lucha por la tierra. Historia de los resguardos del macizo central colombiano. 1944; Bogotá: Punta de Lanza, 1976.

. Bartolomé de Las Casas (1475-1566). Su lucha contra la opresión. Bogotá: Carlos Valencia, 1978.

. Los quimbayas bajo la dominación española. Estudio documental (15391810). Bogotá: Carlos Valencia, 1978.

- El adelantado don Gonzalo Jiménez de Quesada. Bogotá: Carlos Valencia, 1979.

La otra verdad. La Independencia americana vista por los españoles. Bogotá: Carlos Valencia, 1979.

Galvis Madero, Luis. El adelantado. Madrid: Guadarrama, 1957.

Herrera, Antonio de. Historia general de los hechos de los castellanos en las islas y tierra firme del mar océano. 17 t. Madrid: Tipografía de Archivos; Maestre, 1934-1957.

Ibáñez, José María. Ensayo biográfico de Gonzalo Jiménez de Quesada. Bogotá: Imprenta de la Luz, 1892.

Las Casas, Bartolomé de. Historia de las Indias. 3 t. México: Fondo de Cultura Económica, 1951. 
Lucena Salmoral, Manuel. "Epítome de la conquista del Nuevo Reino de Granada, comentario y facsímil”. Ximénez de Quesada (Bogotá), 3, núm. 13 (1962): $33-60$.

- "El indofeudalismo chibcha como explicación de la fácil conquista quesadista”. En: Estudios sobre política indigenista española en América. Vol. 1, 111-160. Valladolid: Universidad de Valladolid, 1975.

. Ximénez de Quesada: el caballero de El Dorado. Madrid: Anaya, 1988.

Maldonado Plata, Joaquín. Gonzalo Jiménez de Quesada. Bogotá: Tipografía Colón, 1913.

Otero D'Costa, Enrique. Gonzalo Jiménez de Quesada. Bogotá: Cromos, 1931.

Oviedo y Valdés, Gonzalo Fernández de. Historia general y natural de las Indias, islas y tierra firme del mar océano. 15 t. [c. 1547]; Asunción, Paraguay: Guarania, 1944.

Piedrahíta, Lucas Fernández de. Historia general de las conquistas del Nuevo Reyno de Granada. 2 t. Amberes, Holanda: Juan Baptista Verdussen, 1688; facsímil, Cali, Colombia: Carvajal, 1986.

Ramos, Demetrio. Ximénez de Quesada en su relación con los cronistas y el epitome de la conquista del Nuevo Reino de Granada. Sevilla: Escuela de Estudios Hispano-Americanos, 1972.

Restrepo, Vicente. Apuntes para la biografía del fundador del Nuevo Reino de Granada, y vidas de los ilustres prelados, hijos de Santafé de Bogotá: Gonzalo Jiménez de Quesada, el Ilustrísimo Sr. D. Hernando Arias de Ugarte, el Ilustrísimo Sr. D. Lucas Fernández Piedrahita. Bogotá: A. M. Silvestre, 1897.

Restrepo Tirado, Ernesto. De Gonzalo Ximénez de Quesada a don Pablo Morillo: documentos inéditos sobre la historia de la Nueva Granada. París: Le Moil, 1928.

Rivas, Raimundo. "Jiménez de Quesada: hombre de Estado". El Gráfico (Bogotá), 12, núm. 609 (1922).

. Los fundadores de Bogotá (Diccionario biográfico). Estudio presentado al Segundo Congreso de Historia y Geografia Hispanoamericana, reunido en Sevilla en mayo de 1921. Bogotá: Imprenta Nacional, 1923. 
Rueda Enciso, José Eduardo. "El pájaro caminante. Biografía de Juan Friede". Mecanografiado. Bogotá, s. f.

Ruiz Rivera, Julián. "De conquistador a colonizador: perfil antiheroico de Jiménez de Quesada". En Memorias del Congreso de Historia del Descubrimiento. Sevilla: 1992, 2:581-582. Sevilla, 1992.

Santos Molano, Enrique. Gonzalo Jiménez de Quesada. Bogotá: Panamericana, 1998.

Sauer, Carl O. "Informe de Carl O. Sauer a la Fundación Rockefeller sobre su visita a Colombia en 1942”. Estudios Sociales (Medellín), 1, núm. 3 (septiembre de 1988): 138-152.

Simón, fray Pedro. Noticias historiales de las conquistas de tierra firme en las Indias Occidentales. 7 t. [¿1628?]; Bogotá: Banco Popular, 1981.

Vallejo, Alejandro. La cita de los aventureros: gesta de don Gonzalo Jiménez de Quesada. Bogotá: Litografía Colombia, 1938.

. La cita de los aventureros: gesta de don Gonzalo Jiménez de Quesada. 1938; Bogotá: Instituto Colombiano de Cultura, 1973.

Fecha de recepción: 23 de agosto de 2005.

Fecha de aceptación: 30 de agosto de 2005. 